\title{
Pregnancy and Covid 19
}

\author{
Kamal el Moussaoui*, Aziz baidada and Aicha Kharbach \\ Department of Gynecology and Obstetrics, Maternity Souissi, University Hospital Center Ibn Sina, Morocco
}

*Corresponding author: Kamal El Moussaoui, Department of Gynecology and Obstetrics, Maternity Souissi, University Hospital Center Ibn Sina, University Mohammed V, Rabat, Morocco

To Cite This Article: Kamal el Moussaoui, Aziz baidada, Aicha Kharbach, Pregnancy and Covid 19. Am J Biomed Sci \& Res. 2021 - 12(2). AJBSR. MS.ID.001739. DOI: 10.34297/AJBSR.2021.12.001739.

Received: 眥 February 02, 2021; Published: 制 March 17, 2021

\begin{abstract}
The COVID19 virus also affects pregnant women. While the vast majority of infected patients have few symptoms, about $10 \%$ of them may have a pneumonia that is important to diagnose and treat. Initial data from the literature suggest management similar to that of the general population. There is a greater risk of premature delivery requiring the administration of corticosteroids for fetal lung maturation in the event of imminent delivery. The route of delivery should be guided by obstetric history even though a large proportion of caesarean sections are included in the published series. Loco-regional anaesthesia is allowed. To date, only a few cases of mother-foetal transmission have been described, rather related to per- or postpartum transmission. The rate of perinatal morbidity and mortality is very low. Only one maternal death has been described. Breastfeeding is allowed with protective measures (hand disinfection, mask for the delivery). In conclusion, all the current studies suggest that pregnant women infected with COVID-19 and their newborn babies have a good prognosis of evolution, but it will be necessary to wait for large multicentric and well-documented series to confirm these data.
\end{abstract}

Keywords: Pregnancy- Covid19

\section{Introduction}

A new strain of coronavirus called SARS (Severe Acute Respiratory Syndrome) -CoV-2 or COVID-19 was isolated from humans in late 2019 in China. She is currently responsible for a pandemic recognized by WHO as a public health emergency. If $75 \%$ are not very symptomatic (flu-like syndrome, myalgia, headache, cough, nasal congestion), the remaining 25\% may present with moderate to severe respiratory distress having taken hospital care and sometimes respiratory assistance. Incubation is on average 5 days (2-14) and contagiousness is greater than that of seasonal influenza. The population particularly at risk of complications concerns elderly, immunocompromised patients or those who carry co-morbid factors such as obesity, cardiovascular disease, diabetes or cancer $[1,2]$. The pregnant woman could theoretically be part of these risk groups by immunosuppression induced by pregnancy as well as other physiological changes such as elevation of the diaphragm, increased oxygen consumption, edema. respiratory mucous membranes which make it more vulnerable to hypoxia [3].

\section{Covid-19 and Pregnancy: What we Know from the Chinese Experience}

Several publications have described the clinical course of pregnant women infected with COVID-19; Chen et al reported 9 cases of pregnant patients who presented with lung disease in the 3rd trimester linked to COVID-19. All of them gave birth by caesarean section either for deterioration of the general condition or because of acute fetal distress. No maternal deaths have been observed. Analysis of amniotic fluid, cord blood, throat smear and breast milk were negative, suggesting the absence of maternalfetal transmission of the virus [4]. Liu et al described a series of 13 pregnant patients between 22 and 36 weeks of which $77 \%$ presented with pyrexia and $3 \%$ were dyspneic. Their clinical course allowed $23 \%$ to return home and the remaining $77 \%$ gave birth by caesarean section either for fetal distress or following a premature rupture of the water bag with failure of the trial of labor. Almost half of them (46\%) gave birth prematurely between 32 and 36 weeks. 
There is one case of fetal death in utero. Only one patient presented with severe respiratory distress requiring the initiation of ECMO (Extracorporeal Membrane Oxygenation) [5].

Chen et al. studied the placentas of 3 pregnant women infected with COVID-19 and who had an emergency cesarean section. Histological analysis showed the presence of fibrin deposits but no lesions suggestive of villitis or chorioamnionitis. Both placentas and newborns have tested negative for the presence of viral RNA suggesting the absence of vertical transmission of COVID-19 [6]. A meta-analysis published by Schwartz on a series of 38 infected Chinese pregnant women shows that COVID-19 causes morbidity and mortality in pregnant women that is less than those of other coronaviruses such as SARS or MERS (Middle east respiratory syndrome). No cases of severe pneumonia or maternal mortality have been reported. However, there is an increased incidence of delivery hemorrhage. There was one neonatal death in a child born prematurely and died on day 9 in a context of multi-systemic decompensation.

Liu et al. reported a series of 15 pregnant women in the third trimester with COVID-19 pneumonia: The main clinical complaints were fever (86\%) and cough (60\%). In biology, lymphopenia was present in $80 \%$ of cases. All patients received a low-dose chest CT scan (between 0.01 and $0.66 \mathrm{mGy}$ ) with a lead abdominal apron that showed images of frosted glass opacities, with peripheral distribution that appear to be pathognomonic for COVID pneumonia. All patients were put on oxygen and none used mechanical ventilation. They did not receive antiviral treatment or hydroxy-chloroquine but only an antibiotic carrier to avoid bacterial superinfection [7]. The latest meta-analysis published by Zaigham and Andersson analysed 108 pregnancies with proven COVID-19 infection, the majority of which were in the Chinese population [8]. In $80 \%$ of cases, the infection occurred in the third trimester, with $68 \%$ having fever, 34\% coughing, 59\% lymphocytopenia and $70 \%$ elevated CRP. A caesarean section was performed in $91 \%$ of cases. With regard to foetal and neonatal morbidity, there was 1 case of foetal death in utero, 1 neonatal death at 34 weeks [9]. In this meta-analysis, it is noted that there were two newborns tested positive for COVID-19 (PCR) and carrying IgM antibodies to COVID, suggesting a possible infection in utero [10]. In the USA, a first series of 43 cases of infected pregnant women reported a rate of $67.4 \%$ of symptomatic patients $(86 \%$ moderate, $9.3 \%$ severe and only $4.7 \%$ critical) Among the $32.6 \%$ of asymptomatic patients detected by universal testing, 70\% later presented symptoms [11].

Finally, a first case of maternal-fetal mortality linked to COVID-19 was reported by Karami et al in Iran in a young 27-yearold patient in the third trimester with severe pneumonia leading to intubation and lethal multisystemic decompensation [12].

\section{Proposed Care For Pregnant Women Infected With Covid-19}

Several consensus articles on the management of pregnant women with a suspected or confirmed diagnosis of VIDOC-19 have been published [13-15].

\section{Prevention}

a. Importance of classic eviction strategies (hand washing, wearing a mask).

b. Since the beginning of April, there has been a recommendation to screen all pregnant women admitted to the delivery room following a New York study. published in the NEJM showing that if $2 \%$ are symptomatic and test positive, there are $13.5 \%$ of parturients who are asymptomatic carriers [16].

\section{Management}

a. In case of suspected infection, if the symptomatology is mild and the patient is not beyond the 24-week term of fetal viability, rest at home and management with the attending physician.

b. If the patient has more severe symptoms (fever, cough, respiratory discomfort) or presents comorbid factors, she should be seen in a COVID screening unit and tested by nasopharyngeal swab (screening for COVID -19 viral RNA and Influenza by RT PCR).

c. In case of suspicion or confirmation of infection, provide for isolation (if possible in a negative pressure room), reception by staff equipped according to recommended standards (FFP2 mask, hat, safety glasses, gown, on shoes).

d. Monitoring of clinical parameters (temperature, pulmonary auscultation, respiratory frequency, 02 saturation) and biological parameters (CRP, haemogram, hepatic enzymology, CK, coagulation tests). Placing an entry route according to severity and if hospitalisation.-Oxygen therapy if Sp02 is less than $95 \%$.

\section{Treatment}

a. Depending on the degree of severity, the various authors propose the use of the same treatments as for other patients.

b. The antiviral drugs of choice during pregnancy are Lopinavir/ritonavir (200mg/50mg per capsule) even if they are not approved by the FDA [14]. Indeed, the risk of teratogenicity of these antivirals is very low [18].

c. Hydroxychloroquine is authorised during pregnancy [15]. 
d. Antibacterial treatment is also recommended to avoid bacterial superinfection, e.g. with IV ceftriaxone, ampicillin or azithromycin $(0.5 \mathrm{mg} / \mathrm{d})$ PO for 5 days [13-15,18].

Corticosteroid use remains a subject of discussion: methylprednisolone (1-2 $\mathrm{mg} / \mathrm{kg} /$ day) has been administered for 3-5 days in cases of severe persistent hypoxemia. If there is a risk of premature delivery, fetal lung maturation with beta or dexamethasone according to standard obstetric protocols should be administered, but in cases of severe or critical impairment, this can be discussed with internists $[15,18]$.

\section{Monitoring of the Foetus}

It should be considered from the time of viability, given the potential risks of fetal distress related to infection and pyrexia. In symptomatic patients and of course, depending on their degree of severity, cardiotoco grams are recommended and should be repeated as well as ultrasound monitoring of fetal growth, amniotic fluid index and fetal Dopplers. Invasive examinations such as amniocentesis are not indicated $[13,15]$.

\section{The Decision to Give Birth}

This should not be taken before term except in the event of a deterioration in maternal health or foetal distress. There appears to be no benefit in recommending a Caesarean section unless the pneumonia is severe and uncontrolled. In the Chinese studies, they were mostly carried out because of a deterioration in foetal cardiac monitoring $[13,15]$. In the case of vaginal delivery, it should be borne in mind that if the amniotic fluid is sterile, the virus may be present in the maternal faeces. The timing of delivery should be chosen if there are associated obstetric complications (placenta previa, pre-eclampsia...) or if the patient's clinical situation remains unstable and not improved by treatment [19].

\section{The Delivery}

It should ideally take place in a room with negative pressure. Staff should be limited to what is strictly necessary and should be equipped with appropriate protective clothing. The route of delivery is related to obstetric conditions, but the risk of Caesarean section must be anticipated to enable the teams to take adequate protective measures in case of emergency [19]. It appears that postpartum haemorrhage is more frequent, as is the case with other infections. In the case of COVID-19 viremia, there are changes in haemostasis parameters which can mimic a HELLP syndrome with thrombocytopenia and hepatic cytolysis. A greater than physiological increase in D-dimers, a drop in fibrinogen, an alteration in PTT and accelerated fibrinolysis can also be observed in cases with a severe prognosis. The thrombotic risk is also greater in patients infected with COVID-19, so care should be taken to ensure proper hydration, use support stockings and administer low molecular weight heparin (LMWH) when bed rest. In the event of Caesarean section, it is recommended that thromboprophylaxis be maintained for 6 weeks postpartum [20]. The use of prostaglandins as a utero tonic should be discussed with internists in view of their broncho constrictor effect in cases of severe pneumonia

\section{Fetal Complications}

To date, no studies have reported cases of fetal malformation or miscarriage associated with COVID-19, but caution should be exercised due to the lack of information on the evolution of infected pregnancies in the first and second trimesters, and one case of in utero mortality in a context of severe maternal respiratory distress and ECMO [5]. There is an increased incidence of premature deliveries, some of which are spontaneous as a result of infection and pyrexia, but others are elective as a result of fetal distress or deterioration in maternal status [4-7]. Given that the pandemic occurred only 4 months ago, there is also a lack of information on the long-term consequences of the inflammation produced after the acute phase of infection on the fetus and placenta. For this reason, there is a need for closer ultrasound follow-up in patients infected during pregnancy, even if they were moderately infected, and for long-term studies of the fate of the children.

\section{Neonatal Complications}

The risk of maternal-fetal vertical transmission appears to be very low and even non-existent in the small series published to date $(4,5,7,8)$ but the whole paragraph will be discussed in another article in this special issue.

\section{Conclusions}

Pregnant women are by definition at greater risk of developing a lung infection mainly due to physiological changes in lung function [3]. All the studies based on small series and meta-analyses show that pregnant women infected with COVID-19 and their newborns have a rather good prognosis both in terms of morbidity and mortality.

\section{Conflict of Interest}

None.

\section{References}

1. G Guan WJ, Ni ZY, Hu Y, Wen-Hua Liang, Chun-Quan Ou, et al. (2020) Clinical Characteristics of Coronavirus Disease 2019 in China. N Engl J Med NEJM oa2002032.

2. Huang C, Wang Y, Li X, Ren L, Zhao J, et al. (2020) Clinical features of patients infected with 2019 novel coronavirus in Wuhan, China. Lancet 395(10223): 497-506.

3. Kaur A, Miller M (2017) General Management Principles of the Pregnant Woman. Semin Respir Crit Care Med 38(2): 123-134.

4. Chen H, Guo J, Wang C, Luo F, Yu X, et al. (2020) Clinical characteristics and intrauterine vertical transmission potential of COVID-19 infection in nine pregnant women: a retrospective review of medical records. Lancet 395(10226): P809-815. 
5. Y Liu, Haihong C, Keijing T, Yubiao G (2020) Clinical manifestations and outcome of SARS-CoV-2 infection during pregnancy. J Infect Mar 4.

6. Chen S, Huang B, Luo DJ, X Li, F Yang, et al. (2020) Pregnant women with new coronavirus infection: a clinical characteristics and placental pathological analysis of three cases. Zhonghua Bing Li Xue Za Zhi 49(0): E005-E05.

7. Schwartz DA (2020) An Analysis of 38 Pregnant Women with COVID-19, Their Newborn Infants, and Maternal-Fetal Transmission of SARSCoV-2: Maternal Coronavirus Infections and Pregnancy Outcomes. Arch Pathol Lab Med 144(7): 799-805.

8. Liu D, Li L, Wu X, Zheng D, Wang J, et al. (2020) Pregnancy and Perinatal Outcomes of Women with Coronavirus Disease (COVID-19) Pneumonia: A Preliminary Analysis. AJR Am J Roentgenol 18:1-6.

9. Zaigham M, Andersson O (2020) Maternal and perinatal outcomes with COVID-19: A systematic review of 108 pregnancies. Acta Obstet Gynecol Scand 99(7):823-829.

10. Zeng H, Xu C, Fan J (2020) Antibodies in Infants Born to Mothers With COVID-19 Pneumonia. JAMA 204861.

11. Breslin N, Baptiste C, Gyamfi-Bannerman C, et al. (2020) COVID-19 infection among asymptomatic and symptomatic pregnant women: Two weeks of confirmed presentations to an affiliated pair of New York City hospitals. Am J Obstet Gynecol MFM 2(2):100118.

12. Karami P, Naghavi M, Feyzi A, Mehdi Aghamohammadi, Mohammad Sadegh Novin, et al. (2020) Mortality of a pregnant patient diagnosed with COVID-19: A case report with clinical, radiological, and histopathological findings. Travel Med Infect Dis 101665.

13. Chen D, Huixia Yang, Yun Cao, Weiwei Cheng, Tao Duan, et al. (2020) Expert consensus for managing pregnant women and neonates born to mothers with suspected or confirmed novel coronavirus (COVID 19) infection. Int J Gynaecol Obstet 149(2):130-136.

14. Rasmussen S, Smulian J, Lednicky J, et al. (2020) Coronavirus Disease 2019 (COVID-19) and Pregnancy: What obstetricians need to know. Am J Obstet Gynecol 222(5):415-426.

15. Peyronnet V, J Sibiude, IP Deruelle, C Huissoud, X Lescure, et al. (2020) Infection par le SARS-CoV-2. Etat des connaissances et proposition de prise en charge. CNGOF. Gynécologie Obstétrique Fertilité \& senologie 48(5): 436-443.

16. Sutton D, Fuchs K, D’Alton M, Goffman D (2020) Universal Screening for SARS-CoV-2 in Women Admitted for Delivery. N Engl J Med 382: 21632164.

17. Moro F, Buonsenso D, Moruzzi MC, R Inchingolo, A Smargiassi, et al. (2020) How to perform lung ultrasound in pregnant women with suspected COVID-19 infection. Ultrasound Obstet Gynecol 55(5): 593598.

18. Liang H, Acharya G (2020) Novel corona virus disease (COVID-19) in pregnancy: What clinical recommendations to follow? Acta Obstet Gynecol Scand 99(4): 439-442.

19. Qi H, Luo X, Zheng Y, Zhang H, Li j, et al. (2020) Safe Delivery for COVID-19 Infected Pregnancies. BJOG 127(8): 927-929.

20. Tao Wang, Ruchong Chen, Chunli Liu, Wenhua Liang, Weijie Guan, et al. (2020) Attention should be paid to venous thromboembolism prophylaxis in the management of COVID-19. Lancet Haematol 7(5): e362-e363. 